\title{
IMPACTO DO PROJETO JOVEM DE FUTURO
}

RICARDO BARROS, MIRELA DE CARVALHO, SAMUEL FRANCO, ANDREZZA ROSALÉM

\section{RESUMO}

Na última década ocorreram progressos significativos em todos os níveis educacionais, sendo particularmente importantes os do ensino médio. 0 Projeto Jovem de Futuro propõe inovar na forma como os serviços vêm sendo ofertados para que a juventude atual possa se beneficiar de uma educação de mais alta qualidade. 0 projeto garante recursos às escolas atendidas, que exercem seu poder discricionário, a fim de superar as limitações mais prementes, e também oferece acesso a tecnologias/metodologias úteis para promover e estimular o aprendizado. Com a criação de um amplo sistema de avaliação de impacto do projeto sobre o aprendizado dos alunos foi possível obter vários resultados importantes, como por exemplo: i) nas escolas beneficiadas os alunos aprenderam quase 60\% mais que os correspondentes alunos nas escolas de controle; ii) os resultados obtidos revelaram uma relação custo-benefício extremamente favorável; e iii) observou-se uma relação positiva entre o desempenho dos alunos e os investimentos em práticas voltadas para aumentar o indice de frequência dos professores. 


\section{RESUMEN}

En la última década ocurrieron progresos significativos en todos los niveles educativos, siendo particularmente importantes los de la enseñanza media. El Proyecto Joven del Futuro propone innovar en la forma como los servicios vienen siendo ofrecidos para que la juventud actual pueda beneficiarse con una educación de más alta calidad. El proyecto garantiza recursos a las escuelas atendidas que ejercen su poder discrecional con el fin de superar las limitaciones más apremiantes y también ofrece el acceso a tecnologías/metodologías útiles para promover y estimular el aprendizaje. Con la creación de un amplio sistema de evaluación de impacto del proyecto sobre el aprendizaje de los alumnos se obtuvieron varios resultados importantes, como por ejemplo: i) en las escuelas beneficiadas los alumnos aprendieron casi un 60\% más que los alumnos de las escuelas de control; ii) los resultados obtenidos revelaron una relación costo-beneficio extremadamente favorable; $y$ iii) se observó una relación positiva entre el desempeño de los alumnos y la inversión en prácticas para aumentar la asistencia de los profesores.

PALABRAS CLAVE: ENSEÑANZA MEDIA • RENDIMIENTO

ESCOLAR • EVALUACIÓN DE LA EDUCACIÓN・PROYECTO JOVEN DEL FUTURO.

\section{ABSTRACT}

In the last decade significant progress has been made on all educational levels, and of particular relevance are those concerning high school. The Future Youth Project proposes to innovate in the way services are being offered so that today's youth can benefit from the highest quality education possible. The project provides resources to those schools addressed, which exercise their discretionary power to overcome their most pressing limitations, and also provides access to useful technologies/methodologies to promote and encourage learning. By creating a comprehensive evaluation system of the project's impact on student learning, it was possible to obtain several important results, such as: i) at the schools addressed students learned almost 60\% more than the corresponding students in control schools; ii) the results revealed an extremely favorable cost-benefit ratio; and iii) there seems to be a positive relation between student performance and investment in increasing the attendance rate of teachers. 


\section{O PROGRESSO RECENTE}

$\mathrm{Na}$ última década ocorreram progressos significativos em todos os níveis educacionais, sendo particularmente importantes os do ensino médio. Por essa razão, nesta nota enfocamos a situação educacional da população em idade de frequentar esse nível de ensino e, portanto, sempre que nos referirmos aos jovens estará compreendido o grupo com idade entre 15 e 17 anos. A porcentagem de jovens na escola aumentou de $76 \%$ em 1998 para 84\% em 2008, enquanto que desses, a porcentagem que estava no ensino médio saltou de menos de $40 \%$ para mais de $60 \%$ no mesmo período.

Por conseguinte, do total de jovens brasileiros, a porcentagem frequentando o ensino médio passou de $30 \%$ para mais de $50 \%$. A taxa de conclusão na idade correta mais do que dobrou, passando de menos de $15 \%$ para mais de $30 \%$.

Mas nem todas as novidades são boas. Uma parte dos jovens ainda está ficando para trás. Quase $10 \%$ dos jovens do país não trabalham nem estudam e mais de $15 \%$ não estudam. Cerca de $40 \%$ dos que estudam ainda estão no fundamental e quase metade dos jovens brasileiros ainda não chegou ao ensino médio. 
O resultado final é que, aos 18 anos, provavelmente mais de 2/3 dos cidadãos ainda não terão completado o ensino médio.

Se os desafios permanecem grandes, ao menos a velocidade de melhora não é baixa, tanto com relação à maior frequência dos jovens à escola como no que diz respeito à redução da defasagem série-idade no fundamental. É de esperar que a matrícula de jovens no ensino médio cresça bastante nos próximos cinco anos.

A crescente valorização do ensino médio encontra pleno suporte no mercado de trabalho. Trabalhadores com essa escolaridade recebem remuneração 11,5\% superior à daqueles com características similares que têm apenas o nível fundamental. Vale notar que o ganho em remuneração para um trabalhador que tenha o ensino fundamental completo vem diminuindo bastante na última década, mas o ganho de ter o ensino médio vem diminuindo menos. Portanto, a evidência indica que o impacto do ensino médio sobre a remuneração deve permanecer importante.

Tão valioso quanto um diploma do ensino médio é o conhecimento adquirido. Estudos recentes (SOARES, 2010) indicam que 10 pontos adicionais de proficiência na escala Saeb elevam em 2,5\% a remuneração do trabalhador. Exatamente por causa dessa substancial valorização por parte do mercado de trabalho, é que deveríamos esperar maiores progressos no sistema de ensino. É surpreendente a ausência de melhora nos indicadores de aprendizado. De fato, segundo o Saeb, tanto em matemática como em língua portuguesa, o desempenho na $3^{\mathrm{a}}$ série do ensino médio piorou entre 1997 e 2007. Igualmente intrigantes são as elevadas taxas de abandono em todas as séries do ensino médio, em particular, na primeira, que no momento corresponde a $15 \%$.

\section{O PROJETO “JOVEM DE FUTURO”}

Porque o mercado de trabalho tem demonstrado valorizar bastante a educação de nível médio e o sistema educacional e a juventude têm respondido com lentidão, fica evidente que existem gargalos nessa oferta. Para que a juventude atual possa se beneficiar de uma educação de mais alta qualidade, é fundamental que existam inovações na forma como os serviços vêm sendo ofertados. O Projeto Jovem de Futuro propõe ser uma dessas inovações. 
A premissa básica é que um dos principais problemas das escolas brasileiras está na falta de protagonismo, isto é, uma ausência de interesse por compreender as limitações existentes e buscar superá-las. Essa falta de protagonismo das escolas decorre, em grande medida, da percepção de que, mesmo quando identificadas suas limitações, ela não disporá dos meios necessários para superá-las.

Por essa razão, o programa garante recursos às escolas atendidas, que exercem seu poder discricionário a fim de superar as limitações mais prementes. Esses recursos, entretanto, são oferecidos sujeitos a uma série de condicionalidades, que buscam adicionalmente incentivar o protagonismo. Tais condicionalidades podem ser de três tipos. Em primeiro lugar, para continuar recebendo recursos do programa, o aprendizado dos alunos precisa melhorar. Portanto, existe um incentivo para que as escolas foquem a atenção no que mais importa: o aprendizado dos alunos. Em segundo lugar, devem realizar um planejamento estratégico participativo, baseado em evidências e voltado para resultados. Por fim, existem restrições quanto ao uso dos recursos, que não podem ser aplicados preponderantemente em infraestrutura, pois há um princípio de que gastos com professores e alunos também devem ser enfocados.

Além de ser uma transferência de recursos com condicionalidades, o projeto também oferece às escolas acesso a tecnologias e metodologias úteis para promover o aprendizado e estimular alunos e professores. Dentre as propostas disponibilizadas, vale chamar a atenção para a formação específica dada aos professores para interpretar o resultado das avaliações dos alunos e reforçar conteúdos ensinados em sala de aula e, do lado dos alunos, o Agente Jovem.

\section{RESULTADOS DA AVALIAÇÃO DE IMPACTO DO PROGRAMA}

Consistente com os princípios de gestão voltada para resultados, foi implantado um amplo e sistemático sistema de avaliação de impacto do projeto sobre o aprendizado dos alunos. A base é a comparação entre o desempenho dos alunos nas escolas selecionadas para participar no programa com o de 
alunos em escolas que demonstraram interesse em participar, mas não foram incluídas nessa fase.

Entre as escolas que declararam interesse em participar, a escolha das beneficiadas foi feita de forma aleatória, garantindo a todas igual chance de participar dessa primeira fase. Caso o programa não tivesse impacto, não haveria razão para o desempenho nas escolas participantes ser distinto do das escolas não participantes. Entretanto, como revela a tabela 1, a proficiência tanto em matemática como em língua portuguesa, após dois anos no programa, é de 15 a 20 pontos na escala Saeb superior nas escolas beneficiadas. Essa é uma estimativa do impacto médio do programa. Conforme ilustra o gráfico 1, nas escolas não beneficiadas de Porto Alegre, a proficiência dos alunos em matemática era 30 pontos na escala Saeb mais elevado ao final da $2^{\mathrm{a}}$ série do ensino médio comparado com o início da $1^{\mathrm{a}}$ série (dois anos antes). Já nas escolas beneficiadas, a proficiência era 47 pontos mais elevada ao final da $2^{\mathrm{a}}$ série comparada ao início da $1^{\text {a }}$ série. Estes resultados indicam que nas escolas beneficiadas, os alunos aprenderam 57\% mais que os correspondentes alunos nas escolas de controle. O impacto do programa foi similar para matemática e língua portuguesa e também de mesma magnitude em Porto Alegre e Belo Horizonte.

TABELA 1 - Estimativas de impacto do Projeto Jovem de Futuro considerando a nota média das escolas nas avaliações de Língua Portuguesa e de Matemática: Porto Alegre e Belo Horizonte, 2008 e 2009

\begin{tabular}{|c|c|c|c|c|c|}
\hline AVALIAÇÕES & $\begin{array}{c}\text { IMPACTO } \\
\text { (PONTOS } \\
\text { NA ESCALA } \\
\text { SAEB) }\end{array}$ & $\begin{array}{l}\text { ERRO-PADRÃO } \\
\text { (PONTOS NA } \\
\text { ESCALA SAEB) }\end{array}$ & $\begin{array}{c}\text { P-VALOR } \\
(\%)\end{array}$ & $\begin{array}{c}\text { PROGRESSO } \\
\text { NA AUSEENCIA } \\
\text { DO PROGRAMA } \\
\text { (PONTOS NA } \\
\text { ESCALA SAEB) }\end{array}$ & $\begin{array}{c}\text { IMPACTO } \\
\text { COMO } \\
\text { PORCENTAGEM } \\
\text { DO } \\
\text { PROGRESSO }\end{array}$ \\
\hline \multicolumn{6}{|l|}{ Porto Alegre } \\
\hline Português & 16 & 6 & 1 & 46 & 34 \\
\hline Matemática & 17 & 5 & 0 & 30 & 55 \\
\hline \multicolumn{6}{|l|}{ Belo Horizonte } \\
\hline Português & 19 & 2 & 0 & 21 & 91 \\
\hline Matemática & 17 & 2 & 0 & 32 & 52 \\
\hline
\end{tabular}

Fonte: Estimativas produzidas com base nas provas Diagnóstica e Somativa do Programa Jovem de Futuro do Instituto Unibanco aplicadas pela Cesgranrio em Porto Alegre e pelo CAED em Belo Horizonte, 2008 e 2009.

Notas:

(1) O impacto foi definido pela diferença do progresso (Somativa - Diagnóstica) das notas médias das escolas de Tratamento e Controle.

(2) O progresso que teria ocorrido na ausência do Programa foi definido pelo progresso (Somativa - Diagnóstica) nas notas médias das escolas de Controle.

(3) Considerou-se como universo todos os alunos que fizeram as avaliações Diagnóstica ou Somativa entre as escolas da amostra. 
GRÁFICO 1 - Evolução da proficiência em matemática: Porto Alegre, avaliações Diagnóstica 2008 e Somativa 2009

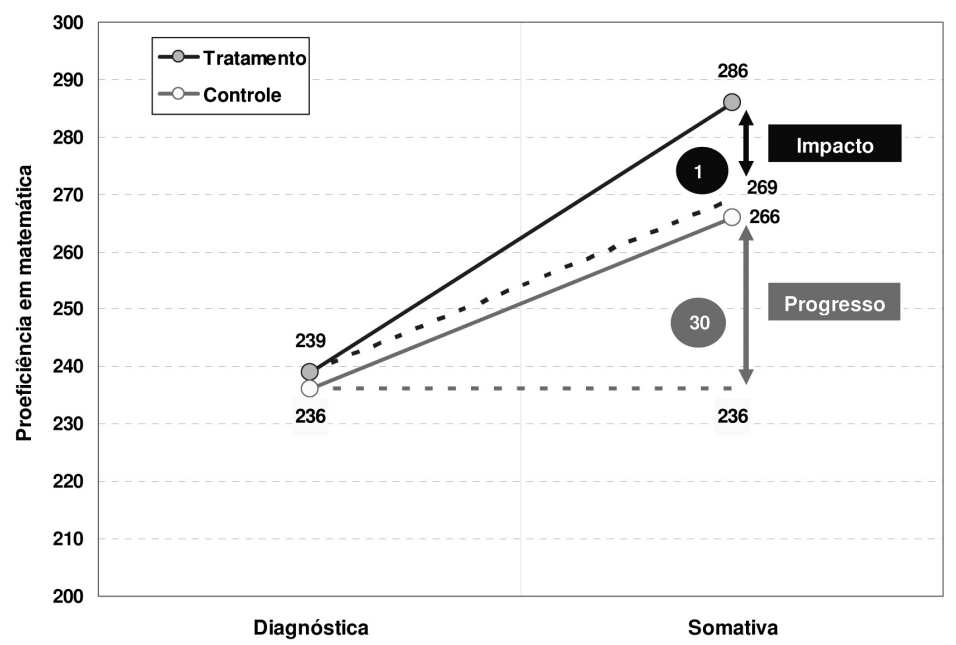

Fonte: Estimativas produzidas com base nas provas Diagnóstica e Somativa do Programa Jovem de Futuro do Instituto Unibanco aplicadas pela Cesgranrio em Porto Alegre, 2008 e 2009.

\section{RELAÇÃO CUSTO-BENEFÍCIO}

Resta avaliar se os benefícios alcançados em termos de maior aprendizado superam os custos incorridos pelo projeto. Conforme já mencionamos, 10 pontos na escala Saeb tendem a elevar a renda do trabalho em $2,5 \%$. Como o impacto do projeto é de no mínimo 15 pontos, ele teria um impacto sobre a renda do trabalho de no mínimo 3,75\%. Para traduzir esse aumento em valores monetários, é necessário estimar o valor presente da renda de um trabalhador típico. Estimativas deste valor para distintas taxas de desemprego e de desconto intertemporal e também diferentes perfis demográficos do trabalhador são apresentadas na tabela 2. Mantidas as condições atuais do mercado de trabalho de Porto Alegre, um trabalhador com ensino médio completo, receberia ao longo de toda a sua vida, uma renda, a valor presente, de no mínimo R\$20 mil. Neste caso, o benefício do programa (3,75\%) para este trabalhador seria um aumento de R\$750 no valor presente de sua renda. Como o custo do programa é no máximo de R\$170 por aluno por ano e, portanto, de R $\$ 340$ por dois anos, o benefício monetário mínimo do programa é mais do que duas vezes seu custo máximo, uma relação custo-benefício, portanto, extremamente favorável. 
TABELA 2 - Avaliação ex-ante do impacto mínimo necessário para uma relação custo-benefício favorável: Porto Alegre, trabalhador com educação média completa

\begin{tabular}{|c|c|c|c|c|c|c|}
\hline GÊNERO & ETNIA & $\begin{array}{c}\text { TAXA DE } \\
\text { EMPREGO } \\
(\%)\end{array}$ & $\begin{array}{c}\text { TAXA DE } \\
\text { DESCONTO } \\
\text { INTERTEMPORAL } \\
(\%)\end{array}$ & $\begin{array}{l}\text { VALOR } \\
\text { PRESENTE DA } \\
\text { RENDA DO } \\
\text { TRABALHO } \\
(\%)\end{array}$ & $\begin{array}{c}\text { BENEFÍCIO } \\
\text { POR UNIDADE } \\
\text { DE IMPACTO } \\
\text { (R\$/PONTOS } \\
\text { NA ESCALA } \\
\text { SAEB) }\end{array}$ & $\begin{array}{c}\text { BENEFÍCIO } \\
\text { CONSIDERANDO } \\
\text { IMPACTO DE } \\
\text { 15 PONTOS NA } \\
\text { ESCALA SAEB } \\
\text { (R\$ MIL) }\end{array}$ \\
\hline \multirow{2}{*}{ Homem } & \multirow{2}{*}{ Branco } & 10 & 10 & 70 & 171 & 2.565 \\
\hline & & 15 & 15 & 34 & 83 & 1.245 \\
\hline \multirow{2}{*}{ Mulher } & \multirow[t]{2}{*}{ Negra } & 10 & 10 & 42 & 103 & 1.545 \\
\hline & & 15 & 15 & 20 & 50 & 0.750 \\
\hline
\end{tabular}

Fonte: Estimativas obtidas com base na Pesquisa Nacional por Amostra de Domicílios (PNAD) de 2008 e no Sistema de Avaliação da Educação Básica (Saeb) de 2005.

Existem, entretanto, incertezas com relação a tal relação. Por um lado, os benefícios podem ser muito maiores se houver crescimento na renda do trabalho. De fato, um crescimento de apenas $1 \%$ na renda do trabalho por ano aumentaria o benefício do programa em 17\%. Também não se está levando em consideração benefícios adicionais, como o aumento que deverá ocorrer na proporção de jovens concluindo o ensino médio e também no acesso à universidade. Por outro lado, o custo por beneficiário pode ser bem mais elevado, dependendo da população que consideremos. O impacto apresentado na tabela 1 foi medido apenas sobre a coorte que iniciava o ensino médio em 2008. Os recursos investidos foram de no máximo $\mathbf{R} \$ 170$ por ano por aluno da escola, incluindo nesse cálculo tanto os alunos nas demais séries do ensino médio, como os que estavam na educação fundamental. Assim, se assumirmos que o benefício do programa foi capturado apenas pelos alunos que estavam na $1^{\text {a }}$ série do ensino médio, então o custo unitário por beneficiário efetivo seria ao menos três vezes mais elevado. Neste caso, o custo poderia chegar a $\mathrm{R} \$ 1.020$ por dois anos de atenção a um beneficiário efetivo, levando a que a relação custo-benefício não seja favorável para um dos grupos considerados: o de mulheres negras com taxas de desemprego e desconto intertemporal de 15\%. Em todos os demais grupos considerados, a relação continua favorável. Vale ressaltar que, nas escolas onde a participação do fundamental no total de matrículas é alta, o custo unitário por beneficiário efetivo seria ainda maior e, portanto, a relação custo-benefício menos favorável. 
Note que, no cenário para homens brancos com taxa de desemprego e de desconto de $10 \%$, a relação custo-benefício seria favorável mesmo que a matrícula total na escola fosse 7 vezes maior que a matrícula na $1^{\mathrm{a}}$ série do médio.

\section{IMPACTO DISTRIBUTIVO}

Em termos ideais, seria desejável que o impacto do projeto fosse maior entre os alunos que inicialmente eram os mais fracos. Os resultados obtidos, entretanto, não apontam para isso.

Conforme a tabela 3 revela, o impacto sobre a proficiência dos $25 \%$ inicialmente piores alunos foi de 11 pontos na escala Saeb, enquanto que sobre os $25 \%$ melhores foi de 16 pontos na escala Saeb. Esse impacto expresso como porcentagem do ganho total de aprendizado de cada grupo é 30\% para os de mais baixo desempenho inicial e de $56 \%$ para os de melhor desempenho inicial.

É importante dizer que, em média, houve uma ligeira redução na desigualdade em proficiência, mas como essa se concentrou mais nas escolas de controle do que nas de tratamento, o projeto não pode ter contribuído para esse feito. De fato, o projeto não parece beneficiar preferencialmente os alunos que partem de patamares mais baixos de proficiência. 


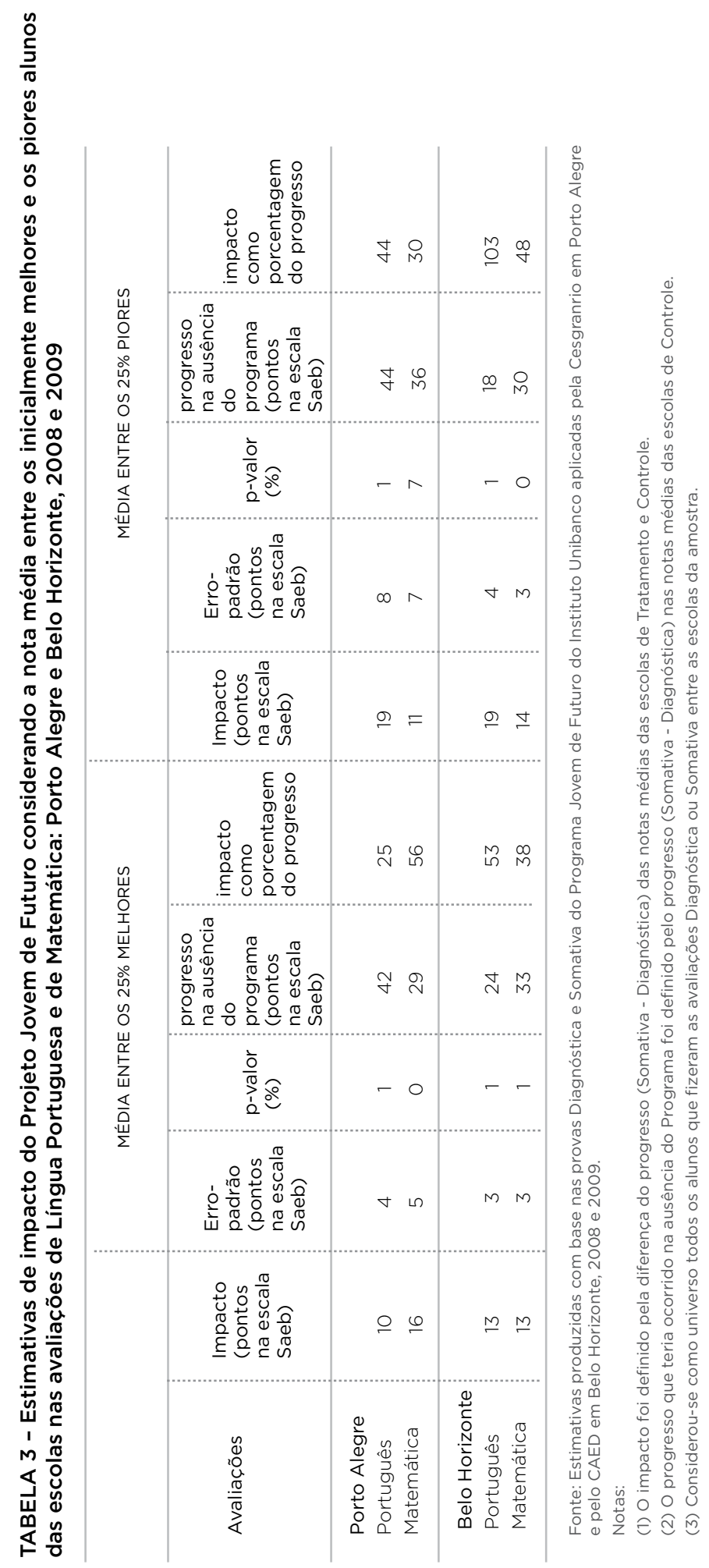


10 desenho do programa nas novas praças para onde se expandiu (escolas na região metropolitana de São Paulo e na Região do Vale do Paraíba) foi alterado. Atualmente o valor total de recursos destinados a cada escola depende do número de alunos no ensino médio, apenas.

\section{FATORES DETERMINANTES DO IMPACTO}

Para o aperfeiçoamento da intervenção, mais importante do que a avaliação geral de impacto, é a análise de como os efeitos finais dependem de diversas características do projeto.

O fato de o projeto garantir considerável liberdade de escolha para que cada escola o implemente de forma diferenciada, oferece à avaliação uma variabilidade extremamente útil para a identificação dos componentes mais importantes a uma maior efetividade.

Ao menos em duas dimensões existe considerável heterogeneidade entre escolas: (a) valor das transferências por aluno na educação média e (b) distribuição dos recursos entre atividades. Como o volume de transferências a cada escola é proporcional à matrícula na escola (aí incluída também a matrícula no ensino fundamental ${ }^{1}$ ) e os gastos, em princípio, deveriam ser com ações direcionadas ao ensino médio, quanto maior a fração da matrícula da escola na educação fundamental, maior deverá ser a transferência por aluno no ensino médio. Assim, se o volume de recursos é um importante determinante do impacto, deveríamos observar melhores resultados nas escolas com maior participação do fundamental no total de matrículas.

A análise das informações disponíveis não revela, entretanto, qualquer associação estatisticamente significativa entre o impacto do programa e a proporção da matrícula no fundamental. Este resultado deve ser tratado com extrema cautela, pois as escolas com maior participação do fundamental na matrícula diferem das demais por inúmeras razões que não estão sendo consideradas e que também podem influenciar o impacto do programa.

No que se refere à distribuição dos recursos por tipo de atividade, parece existir uma correlação positiva entre o desempenho dos alunos e os investimentos em práticas voltadas para aumentar o índice de frequência dos professores (Gráfico 2). 
GRÁFICO 2 - Relação entre o impacto do programa e a proporção dos recursos alocados a atividades que promovam o aumento do índice de frequência dos professores: Língua portuguesa - Porto Alegre, 2008

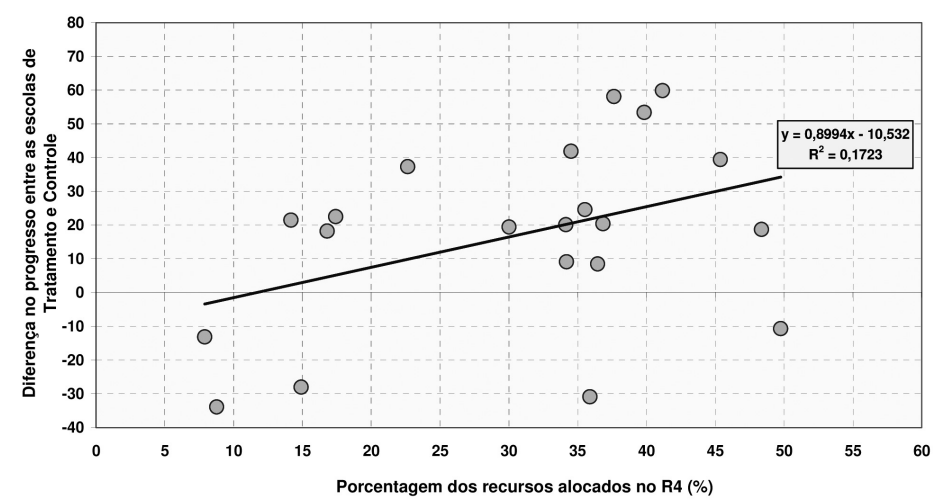

Fonte: Estimativas produzidas com base nas provas Diagnóstica e Somativa do Programa Jovem de Futuro do Instituto Unibanco aplicadas pela Cesgranrio em Porto Alegre, 2008 e 2009. As informações da alocação de recursos do programa por escola foram disponibilizadas pelo Instituto Unibanco para anos de 2008 e 2009.

Nota 1: Foi considerado como impacto a diferença na nota média no progresso (Somativa 2009 - Diagnóstica 2008) entre as escolas de Tratamento e Controle.

\section{REFERÊNCIAS BIBLIOGRÁFICAS}

SOARES, S. O Conhecimento paga bem? Habilidades cognitivas e rendimentos do trabalho no Brasil (e no Chile). 2010. Tese (Doutorado) Universidade de Brasília (UnB). Brasília, 2010.

\section{RICARDO BARROS}

Subsecretário da Secretaria de Assuntos Estratégicos da Presidência da República ricardo.barros@presidencia.gov.br

\section{MIRELA DE CARVALHO}

Assessora Especial da Secretaria de Estado de Educação do Rio de Janeiro mirela.carvalho@educacao.rj.gov.br 


\section{SAMUEL FRANCO}

Estatístico, consultor associado do Instituto de Estudos do Trabalho e Sociedade (IETS)

samolivafranco@gmail.com

\section{ANDREZZA ROSALÉM}

Economista, consultora associada do Instituto de Estudos do Trabalho e Sociedade (IETS)

andrezzarosalem@gmail.com 